Effect of magnetic geometry on transport of neutral particles locally entering confined plasma volume

M. Z. Tokar

Citation: Physics of Plasmas 16, 094506 (2009); doi: 10.1063/1.3236398

View online: https://doi.org/10.1063/1.3236398

View Table of Contents: http://aip.scitation.org/toc/php/16/9

Published by the American Institute of Physics

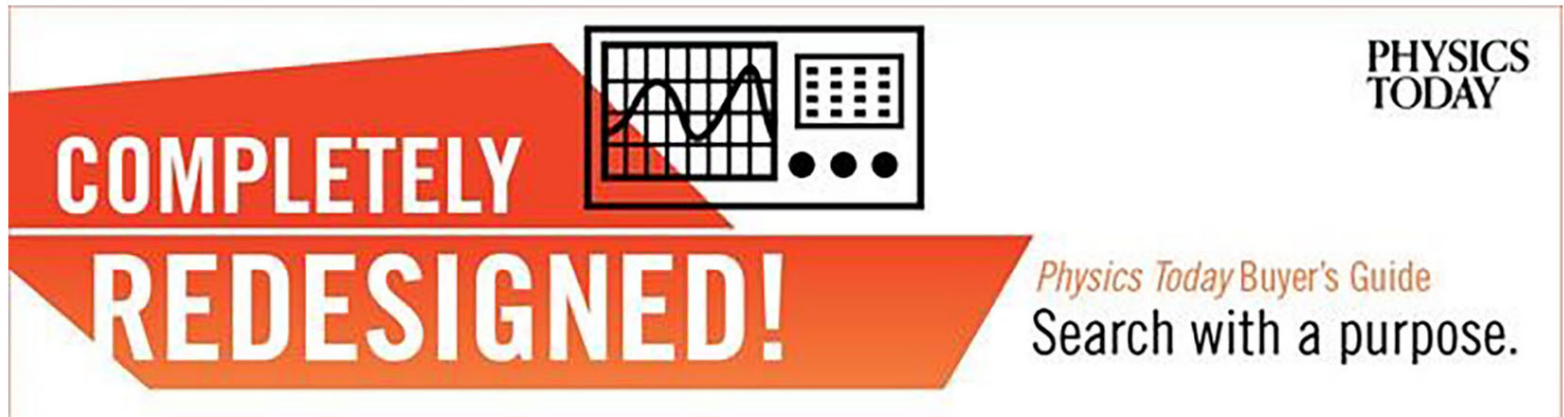




\title{
Effect of magnetic geometry on transport of neutral particles locally entering confined plasma volume
}

\author{
M. Z. Tokar \\ Institut für Energieforschung-Plasmaphysik, Forschungszentrum Jülich GmbH, \\ Association FZJ-Euratom, 52425 Jülich, Germany
}

(Received 30 June 2009; accepted 2 September 2009; published online 21 September 2009)

\begin{abstract}
The effect of the position where neutral particles enter the confined plasma region with closed magnetic surfaces on the radial profiles of the neutral flux and plasma density is numerically investigated. The case of elongated magnetic surfaces with $X$-point, in whose vicinity the metric coefficients are varying very fast with the radius of magnetic surfaces, is considered. (C) 2009 American Institute of Physics. [doi:10.1063/1.3236398]
\end{abstract}

Neutral particles, e.g., released from the wall elements or injected by gas puffing through special valves, are important constituents of fusion plasmas. The ionization of neutrals by electrons, charge exchange in collisions with ions, and diverse other processes are of principal importance for the generation of charged particles, losses of thermal energy, and therefore, essentially determine the densities and temperatures of plasma components. ${ }^{1}$ Thus, in the divertor volume neutral particles principally control the conditions for plasma detachment from the target plates, the most acceptable operational regime for ITER. ${ }^{2}$ Here, we consider neutrals that penetrate through the separatrix into confined plasma volume with closed magnetic surfaces. Their ionization affects the radial gradient of the plasma density $n$ and can play an important role for the formation of the edge transport barrier (ETB) in the $H$-mode. ${ }^{3}$ The closeness of the neutral penetration depth to the width of the ETB in the density profile, $\Delta_{n}$, has been found on the tokamak DIII-D. ${ }^{4,5}$ This is in agreement with a simple neutral penetration model (see, e.g., Refs. 6 and 7), predicting $\Delta_{n} \sim 1 / n_{\mathrm{PED}}$, where $n_{\mathrm{PED}}$ is the pedestal density. A weaker correlation between these parameters has been, however, seen in the Alcator C-Mod machine. ${ }^{8}$ The radial inhomogeneity of the diffusivity of charged particles in the ETB and the velocity distribution of neutral particles have been invoked to explain these findings. ${ }^{8}$ In addition to the above mentioned factors, also peculiarities of the magnetic geometry, such as elongation of magnetic surfaces, can affect the transport of neutral particles significantly. As it has been shown in Refs. 4 and 9, $\Delta_{n}$ varies inversely proportional to the flux expansion parameter taken at the poloidal position of the neutral injection. An essential assumption made in Refs. 4 and 9 is a weak variation in this characteristic with the minor radius of magnetic surfaces. This supposition fails, however, if neutrals enter the plasma near an $X$-point. In such a case, an analytical consideration performed in Refs. 4 and 9 is unsure and a numerical solution of transport equations for neutral and charged particles is necessary. This is the main objective of the present paper. The approach elaborated can be straightforwardly incorporated in one-dimensional transport codes used for calculations of radial profiles of plasma parameters inside the separatrix. ${ }^{10-14}$

Although Monte Carlo methods ${ }^{15}$ are more proper by considering the transfer of neutral particles in complex geometries, an approximate fluid description is applied in this study to get a more physical insight into the problem. Neutral-neutral collisions and plasma bulk velocity are henceforth neglected. Consider neutral atoms with a prescribed temperature $T_{01}$ which enter very locally, through the separatrix, the confined volume of a hydrogen isotope plasma (see Fig. 1). These "primary" neutrals are ionized by electrons and charge exchange with ions, with the rate coefficients $k_{\text {ion }}$ and $k_{\mathrm{cx}}$, respectively. Their total disintegration frequency is equal to $\nu_{n}=\nu_{\text {ion }}+\nu_{\mathrm{cx}}$, with $\nu_{\text {ion }}=k_{\text {ion }} n$ and $n u_{\mathrm{cx}}=k_{\mathrm{cx}} n$. In a fluid approximation (see, e.g., Ref. 16), the particle density $n_{01}$ and flux density $\mathbf{j}_{01}$ of primary neutrals are governed by the following continuity and force balance equations:

$$
\begin{aligned}
& \nabla \cdot \mathbf{j}_{01} \equiv \frac{\partial j_{01, \xi}}{\partial \xi}+\nabla_{\chi} \cdot \mathbf{j}_{01, \chi}=-\nu_{n} n_{01}, \\
& \frac{\partial}{\partial \xi}\left(n_{01} T_{01}\right)=-m \nu_{n} j_{01, \xi},
\end{aligned}
$$

where $\xi$ and $\chi$ are the directions normal and tangent to the magnetic surface, correspondingly, $j_{01, \xi}$ and $\mathbf{j}_{01, \chi}$ are the respective components of the flux density, and $m$ is the atom mass equal to that of plasma ions. For realistic $n$, the penetration depth of neutral particles in the plasma until ionization is much less than the device dimensions. Thus, if the position of the neutral entrance into the confined volume is strongly localized, then the cross sections of the neutral cloud by magnetic surfaces are much smaller than the total surface area. In such a case, it is convenient to proceed to the total flux and the total number of neutral particles in such a cross section,

$$
\left(\begin{array}{c}
J_{01} \\
N_{01}
\end{array}\right)=\int\left(\begin{array}{c}
j_{01, \xi} \\
n_{01}
\end{array}\right) d S_{\chi}
$$

At the boundaries of this area $\mathbf{j}_{01, \chi} \rightarrow 0$ and by integrating Eqs. (1) and (2), we get the following equations describing the variation in $J_{01}$ and $N_{01}$ with $\xi$ : 


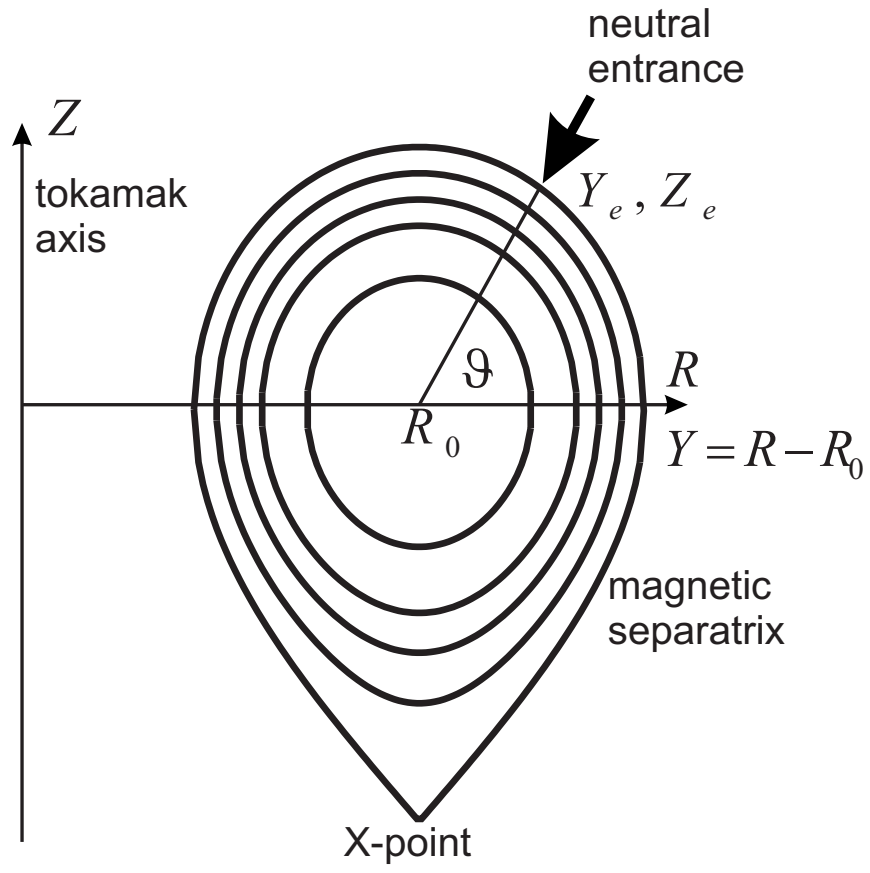

FIG. 1. The poloidal cross section of closed elongated magnetic surfaces in a tokamak with $X$-point. The entrance of neutral particles through the magnetic separatrix is localized at the poloidal angle $\vartheta$, in the point with the Cartesian coordinates $Y_{e}$ and $Z_{e}$.

$$
\frac{d J_{01}}{d \xi}=-\nu_{n} N_{01}, \quad \frac{d}{d \xi}\left(N_{01} T_{01}\right)=-m \nu_{n} J_{01}
$$

In this study, it is assumed that the plasma parameters and, thus, $\nu_{n}$ are constant on magnetic surfaces labeled by the index $\rho$. In particular, the effects due to local plasma cooling through energy losses by neutral ionization are neglected. This is acceptable if the plasma density is not too high. ${ }^{6,17}$ In such a case, Eq. (4) can be combined in the following one, which describes the $\rho$-dependence of $J_{01}$ :

$$
\frac{d}{d \rho}\left(\frac{1}{g \nu_{n}} \frac{d J_{01}}{d \rho}\right)=m g \frac{\nu_{n}}{T_{01}} J_{01}
$$

Here, the metric coefficient $g=d \xi / d \rho$ is determined by the geometry of magnetic surfaces and depends on the position where neutrals enter through the separatrix. The boundary conditions to Eq. (5) are adopted as follows: $J_{01}$ reduces to zero at the plasma axis, $\rho=0$, and the total influx of neutrals through the separatrix, $-J_{01}(\rho=a)$, is prescribed.

The charge exchange of primary neutrals with ions generate "secondary" atoms with the local ion temperature and averaged velocity assumed zero. These atoms move a finite distance perpendicular to the magnetic surfaces before the next charge exchange or ionization takes place. Therefore, at a given point, particles generated at different positions are present and their averaged energy is not defined by the local ion temperature $T_{i}$ only. Thus, in addition to the continuity and momentum equations:

$$
\nabla \cdot \mathbf{j}_{02}=\nu_{\mathrm{cx}} n_{01}-\nu_{\mathrm{ion}} n_{02}
$$

$$
\frac{d}{d \xi}\left(n_{02} T_{02}\right)=-m \nu_{n} j_{02, \xi}
$$

for the particle density $n_{02}$ and flux density $\mathbf{j}_{02}$, the temperature $T_{02}$ of the secondary atoms has to be determined from the heat balance equation:

$$
\nabla \cdot\left(\frac{3}{2} T_{02} \mathbf{j}_{02}\right)=\frac{3}{2} T_{i} \nu_{\mathrm{cx}} n_{01}-\frac{3}{2} T_{02} \nu_{\text {ion }} n_{02} .
$$

As in the case of primary neutrals, we introduce integrated variables,

$$
\left(\begin{array}{c}
J_{02} \\
P_{02}
\end{array}\right)=\int\left(\begin{array}{c}
j_{02, \xi} \\
n_{02} T_{02}
\end{array}\right) d S_{\chi}
$$

The procedure analogous to that applied above results in the following equation for $P_{02}$ :

$$
\frac{d}{d \rho}\left(\frac{1}{g \nu_{n}} \frac{d P_{02}}{d \rho}\right)=m g\left(\frac{\nu_{\text {ion }}}{T_{02}} P_{02}-\nu_{\mathrm{cx}} N_{01}\right) .
$$

The boundary conditions to this are governed by the relationship,

$$
J_{02}=-\frac{1}{m g \nu_{n}} \frac{d P_{02}}{d \rho}
$$

following from Eq. (7). Thus, zero flux at the plasma axis results in $d P_{02} / d \rho=0$; the secondary neutrals escape from the plasma through the separatrix with their thermal velocity $V_{02}=\sqrt{T_{02} / m}$, and this provides

$$
P_{02}+\frac{V_{02}}{g \nu_{n}} \frac{d P_{02}}{d \rho}=0
$$

Equations (5) and (10) are solved numerically by the method described in Ref. 18. Equation (8) is reduced to the following one:

$$
\frac{d T_{02}}{d \rho}=\frac{g \nu_{\mathrm{cx}} N_{01}}{J_{02}}\left(T_{i}-T_{02}\right)
$$

which is treated by a technique employed in the RITM code. $^{14,19}$ The source of secondary neutrals is located inside the plasma and they move toward both the core and the separatrix. Thus, there is a point $\rho_{*}$ where $J_{02}=0$ and $T_{02}=T_{i}$ because $d T_{02} / d \rho$ has to be finite here. With known $T_{02}\left(\rho_{*}\right)=T_{i}\left(\rho_{*}\right)$, Eq. (13) can be integrated from $\rho_{*}$ in both directions, toward $\rho=0$ and $\rho=a$.

For a distributed influx of primary neutrals through the separatrix, with the density $j_{01, \xi}(a, l)$ being a smooth function of the distance $l$ on the magnetic surface in the poloidal direction counted from the meridional plane, one can consider the total neutral population as a superposition of localized clouds described above. In the first approximation, those are independent in the sense that particles from different clouds are not mixed up. Each cloud is characterized by the total influx $d J_{01}(a, l)=j_{01, \xi}(a, l) d l$.

In a stationary state there is a balance between the total influx of neutrals and the outflow of charged particles inside any closed magnetic surface. Henceforth, we assume that the plasma particle transfer is due to diffusion with the coeffi- 


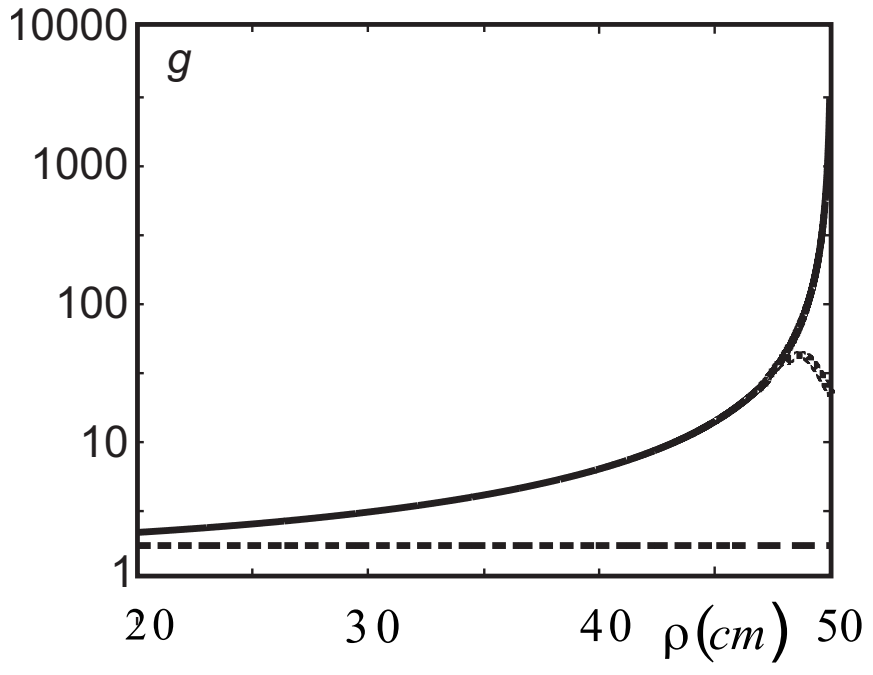

FIG. 2. The $\rho$-profiles of the metric coefficient $g$ for the entrance of neutrals at the top, $\vartheta=\pi / 2$ (dashed line), and close to the $X$-point at $\vartheta=-\pi / 2+0.1$ (dotted curve) and at $\vartheta=-\pi / 2+0.01$ (solid curve).

cient $D$ and flux density $-D d n / d s \equiv-D / g \times d n / d \rho$. Thus, for $n$ homogeneous on magnetic surfaces, the particle balance is given by

$$
-D \frac{d n}{d \rho} \cdot 2 \pi R_{0} \oint \frac{d l}{g(\rho, l)}+J_{01}(\rho)+J_{02}(\rho)=0,
$$

where $R_{0}$ is the major radius of the torus. Here and henceforth any effects of the toroidal geometry are neglected. As a boundary condition the density $e$-folding length $\delta$ is prescribed at the separatrix: $d n / d \rho=-n / \delta$. With a given $D(\rho)$, a converged solution of the system of nonlinear differential equations (5), (10), (13), and (14) is found in an iterative procedure.

The cross section of magnetic surfaces under consideration is displayed in Fig. 1. Its upper part at $Z \geq 0$ is compounded of half-ellipses given by the equation,

$$
Y^{2}+Z^{2} / E^{2}=\rho^{2},
$$

where $E$ is the elongation and $0 \leq \rho \leq a$ is the half-width of the ellipse. The entrance of neutrals is located at the poloidal angle $\vartheta$ and has the Cartesian coordinates:

$$
Y_{e}=\frac{a}{\sqrt{1+(\tan \vartheta / E)^{2}}}, \quad Z_{e}=Y_{e} \tan \vartheta .
$$

By integrating the tangent equation $d Y / d Z=-Z /\left(Y E^{2}\right)$ with these initial conditions, one gets $Y=Y_{e}\left(Z / Z_{e}\right)^{E^{2}}$. The intersection of the tangent with magnetic surface of any particular $\rho$ provides a transcendent algebraic equation for $Z(\vartheta, \rho)$. With known $Y$ and $Z$, the metric coefficient is calculated as follows:

$$
g=\frac{d Z}{d \rho} \sqrt{1+\left(\frac{d Y}{d Z}\right)^{2}}=\frac{\rho}{\sqrt{Y^{2}+Z^{2} / E^{4}}} .
$$

In the lower part, $Z \leq 0$, there is an $X$-point and the magnetic half-surfaces are approximated by the equation,

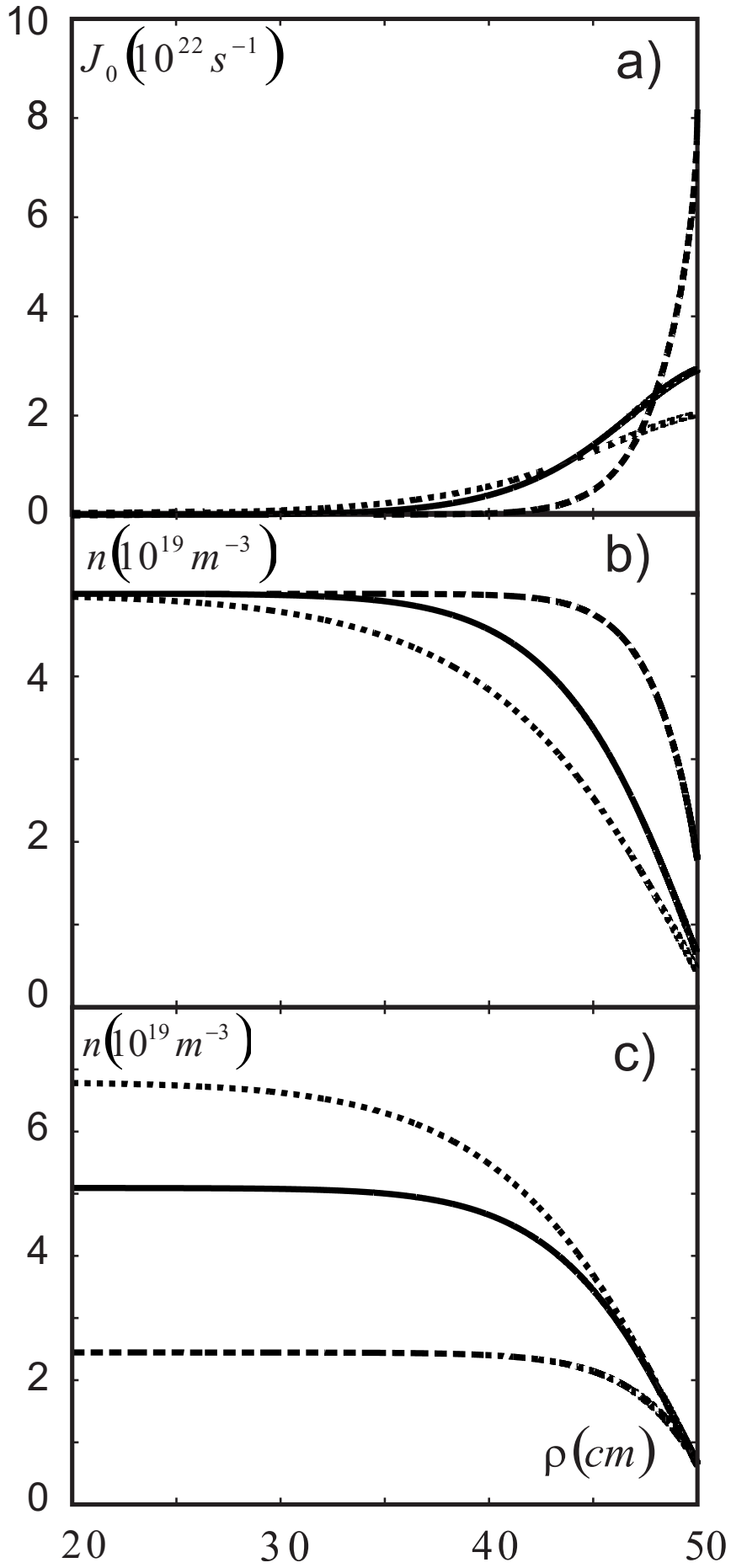

FIG. 3. The $\rho$-profiles of the total influx of neutral particles and plasma density computed with [(a) and (b) $n(0)=5 \times 10^{19} \mathrm{~m}^{-3}$ and with (c) $-J_{01}(\rho=a)=3 \times 10^{22} \mathrm{~s}^{-1}$ for different positions of the neutral entrance through the separatrix: at the top, $\vartheta=\pi / 2$ (solid lines), at the equatorial plane, $\vartheta=0$ (dotted lines), and close to the $X$-point, $\vartheta=-\pi / 2+0.01$ (dashed lines).

$$
Y^{2}+a^{2} \sin ^{2}\left(\frac{Z}{a E}\right)=\rho^{2},
$$

which reduces to Eq. (15) for $\rho \ll a$. In this case the neutral injection position on the separatrix at the poloidal angle $\vartheta$ has Cartesian coordinates which have to be determined by solving the equations $Y_{e}=a \cos \left[Z_{e} /(a E)\right]$ and $Z_{e}=Y_{e} \tan \vartheta$. 
The tangent, beginning at this position, is given by the relation

$$
Y=Y_{e}\left[\tan \left(\frac{Z}{a E}\right) / \tan \left(\frac{Z_{e}}{a E}\right)\right]^{E^{2}}
$$

and

$$
g=\frac{\rho}{\sqrt{Y^{2}+\frac{a^{2}}{4 E^{2}} \sin ^{2}\left(\frac{2 Z}{a E}\right)}} .
$$

For the length element on the magnetic surface in the poloidal direction, one has $d l=\rho /(g Y) \times d Z$.

Calculations have been done for a medium sized divertor tokamak such as ASDEX-Upgrade with the parameters $a=0.5 \mathrm{~m}, R_{0}=1.65 \mathrm{~m}$, and $E=1.7 .^{20}$ The radial variation in the metric coefficient $g$ calculated for different positions of the neutral injection is shown in Fig. 2. When the injection position is close to the $X$-point, it becomes very fast by approaching to the separatrix. The rate coefficients for ionization and charge exchange are taken from Ref. 14. A Gaussian profile $T(0) \exp \left(-\rho^{2} / a_{T}^{2}\right)$, with $T(0)=1.5 \mathrm{keV}$ and $a_{T}=a / 1.7$, is assumed identically for temperatures of electrons and ions; the temperature of primary neutrals $T_{01}=T(a)$, their total influx $\Phi_{0}$ is selected in an iterative procedure to reproduce the given value of the central plasma density, $n(\rho=0)=5 \times 10^{19} \mathrm{~m}^{-3}$; the plasma density $e$-folding length at the separatrix $\delta=1 \mathrm{~cm}$, and the diffusivity of charged particles $D(\rho) \equiv 1 \mathrm{~m}^{2} / \mathrm{s}$.

Figure 3(a) shows the $\rho$-profiles of the total neutral influx, $J_{0}=-J_{01}-J_{02}$, which is equal in a steady state to the plasma outflow, found with several positions of the neutral entrance: at the outboard, $\vartheta=0$, at the top, $\vartheta=\pi / 2$, and close to the $X$-point, $\vartheta=-\pi / 2+0.01$. Calculation with $\vartheta=-\pi / 2$ exactly is not possible because $g$ is undefined at the separatrix in this case. One can see that the penetration depth in the $\rho$-units decreases significantly by changing the entrance position from the equatorial plane to the top of the elongated half-surface and, finally, into the vicinity of the $X$-point. This is explained by the fact, already noticed in Refs. 4 and 9, that the physical distance passed by a neutral in the plasma, $d s=g \cdot d \rho$, increases with the metric coefficient $g$ which grows up for elongated plasmas with $E>1$ when going toward the vertical axis at $Y=0$; by approaching the $X$-point, $g$ rises even substantially stronger and is very $\rho$-dependent. The calculated profiles of the plasma density are shown in Fig. 3(b). Corresponding to the neutral influx, also the gradient region on the density profile becomes much narrower with increasing flux expansion factor $g$. In the case of dotted and solid curves with $g$ independent of $\rho$ and equal to 1 and $E$, correspondingly, the found profiles can be well approximated by the analytical relations from Refs. 4 and 9. For neutral injection near the $X$-point, see the dashed curve, $g$ is very strong function of the radius and any analytical assessment of the $n(\rho)$ dependence is obsolete. Since in the steady state the density gradient defines both the outflow of plasma particles and the influx of neutrals, this explains the much larger value of the neutral influx at the separatrix required for the adopted $n(\rho=0)$.
Finally, we consider a situation with a given total influx of primary neutrals through the separatrix, $-J_{01}(\rho=a)=3$ $\times 10^{22} \mathrm{~s}^{-1}$. The stationary profiles of the plasma density in this case are shown in Fig. 3(c). The gradient part of the density profile is not generally narrowing with increasing central density as it is usually expected because the neutral penetration depth is inversely proportional to $n$. For constant $g(\rho)$, the found profiles can be well described by analytical results from Refs. 4 and 9. This approach is not, however, appropriate if neutrals enter the confined volume near the $X$-point.

Thus the position where neutrals enter the confined volume through the separatrix significantly affects the neutral transport and is very important for the resulting plasma density profile. This fact has to be properly taken into account in transport modeling of the plasma interior with onedimensional codes. It may also be of relevance to the important phenomenon of density limit at which the divertor region is cooled down and more neutrals escape into the confined plasma near the $X$-point. This will be studied in detail elsewhere.

${ }^{1}$ P. C. Stangeby, The Plasma Boundary of Magnetic Fusion Device (IoP, Bristol, 2000), p. 22.

${ }^{2}$ A. S. Kukushkin, H. D. Pacher, V. Kotov, D. Reiter, D. Coster, and G. W. Pacher, Nucl. Fusion 45, 608 (2005).

${ }^{3}$ F. L. Hinton and G. M. Staebler, Phys. Fluids B 5, 1281 (1993).

${ }^{4}$ M. A. Mahdavi, R. Maingi, R. J. Groebner, A. W. Leonard, T. H. Osborne, and G. Porter, Phys. Plasmas 10, 3984 (2003).

${ }^{5}$ R. J. Groebner, M. A. Mahdavi, A. W. Leonard, T. H. Osborne, N. S. Wolf, G. D. Porter, P. C. Stangeby, N. S. Brooks, R. J. Colchin, and L. W. Owen, Nucl. Fusion 44, 204 (2004).

${ }^{6}$ M. Z. Tokar, Plasma Phys. Controlled Fusion 35, 1119 (1993).

${ }^{7}$ P. C. Stangeby, J. Phys. D: Appl. Phys. 36, 2784 (2003).

${ }^{8}$ J. W. Hughes, B. LaBombard, D. A. Mossessian, A. E. Hubbard, J. Terry, T. Biewer, and Alcator C-Mod Team, Phys. Plasmas 13, 056103 (2006).

${ }^{9}$ M. A. Mahdavi, T. H. Osborne, A. W. Leonard, M. S. Chu, E. J. Doyle, M. E. Fenstermacher, G. R. McKee, G. M. Staebler, T. W. Petrie, M. R. Wade, S. L. Allen, J. A. Boedo, N. H. Brooks, R. J. Colchin, T. E. Evans, C. M. Greenfield, G. D. Porter, R. C. Isler, R. J. La Haye, C. J. Lasnier, R. Maingi, R. A. Moyer, M. J. Schaffer, P. G. Stangeby, J. G. Watkins, W. P. West, D. G. Whyte, and N. S. Wolf, Nucl. Fusion 42, 52 (2002).

${ }^{10}$ C. E. Singer, D. E. Post, D. R. Mikkelsen, M. H. Redi, A. McKenney, A. Silverman, F. G. P. Seidl, P. H. Rutherford, R. J. Hawryluk, W. D. Langer, L. Foote, D. B. Heifetz, W. A. Houlberg, M. H. Hughes, R. V. Jensen, G. Lister, and J. Ogden, Comput. Phys. Commun. 49, 275 (1988).

${ }^{11}$ A. Airoldi and G. Cenacchi, Nucl. Fusion 37, 1117 (1997).

${ }^{12}$ ASTRA online manual: http://www.ipp.mpg.de/ git/astra/ section.php?sec $=0$.

${ }^{13}$ V. Basiuk, J. F. Artaud, F. Imbeaux, X. Litaudon, A. Becoulet, L.-G. Eriksson, G. T. Hoang, G. Huysmans, D. Mazon, D. Moreau, and Y. Peysson, Nucl. Fusion 43, 822 (2003).

${ }^{14}$ M. Z. Tokar', Plasma Phys. Controlled Fusion 36, 1819 (1994).

${ }^{15}$ D. Reiter, V. Kotov, P. Börner, K. Sawada, R. K. Janev, and B. Küppers, J. Nucl. Mater. 363-365, 649 (2007).

${ }^{16}$ T. D. Rognlien, P. N. Brown, R. B. Campbell, T. B. Kaiser, D. A. Knoll, P. R. McHugh, G. D. Porter, M. E. Rensink, and G. R. Smith, Contrib. Plasma Phys. 34, 362 (1994).

${ }^{17}$ D. Löchel, M. Z. Tokar, M. Hochbruck, and D. Reiser, Phys. Plasmas 16, 044508 (2009).

${ }^{18}$ M. Z. Tokar, D. Kalupin, and D. Pilipenko, Comput. Phys. Commun. 175, 30 (2006).

${ }^{19}$ D. Pilipenko, M. Z. Tokar, I. Pavlenko, and B. Weyssow, Plasma Phys. 12, 072521 (2005).

${ }^{20}$ J. Wesson, Tokamaks, 3rd ed. (Clarendon, Oxford, 2004), p. 149. 